\title{
AFECTACIONES FINANCIERAS, COMPETITIVAS Y ECONÓMICAS DEL SECTOR AZUCARERO COLOMBIANO RESPECTO A LA NORMATIVIDAD COMERCIAL, FISCAL Y LABORAL ${ }^{\star}$
}

\section{FINANCIAL, COMPETITIVE AND ECONOMICS EFFECTS OF THE COLOMBIAN SUGAR SECTOR WITH RESPECT TO COMMERCIAL, FISCAL AND LABOR REGULATIONS}

Recibido: 4 de abril de 2019

Evaluado: 1 de mayo de 2019

Aprobado: 5 de junio de 2019

\author{
Martha Lucía Fuertes Díaz* \\ Universidad Nacional Abierta y a Distancia, UNAD \\ Orcid: 0000-0001-8468-1867 \\ Julio Cesar Montoya Rendón ${ }^{* \star *}$ \\ Universidad Nacional Abierta y a Distancia, UNAD \\ Orcid: 0000-0003-1170-9296
}

Cómo citar este artículo: Fuertes Díaz, M.I. y Montoya Rendón, J. C. (2019). Afectaciones financieras, competitivas y económicas del sector azucarero colombiano respecto a la normatividad comercial, fiscal y laboral. Revista Estrategia Organizacional, 8 (2), pp. $75-101$. doi: https://doi.org/10.22490/25392786.3433

* $\quad$ Artículo de investigación.

** Doctora en Administración Gerencial, Universidad Benito Juárez, México. Magíster en Administración con énfasis en Finanzas Corporativas, Universidad ICESI, Colombia. Especialista en Finanzas con Concentración en Finanzas Avanzadas, Universidad ICESI, Colombia. Contadora Pública, Universidad Santiago de Cali, Colombia. Docente Universidad Nacional Abierta y a Distancia, UNAD, Colombia. Investigadora Grupo de Investigación ilama. Correo electrónico: martha.fuertes@unad.edu.co

*** Magister en Administración, Especialista en Pedagogía para el Desarrollo del Aprendizaje Autónomo, Administrador de Empresas. Docente Universidad Nacional Abierta y a Distancia, UNAD, Colombia. Investigador del grupo ilama. 


\title{
RESUMEN
}

Esta investigación analiza el avance en materia de competitividad y desempeño financiero del sector azucarero colombiano durante el período comprendido entre los años 2013 a 2016, en contraste con las políticas públicas emanadas en materia comercial, laboral y fiscal y las posibles afectaciones para su desempeño. Se sustenta a partir de las teorías de recursos y capacidades y desde las finanzas corporativas con los indicadores financieros de rentabilidad para medir la efectividad empresarial al asociar la gestión en costos y gastos para la generación de beneficioses. La investigación es descriptiva, longitudinal, e inductiva y utiliza el software estadístico SAS para el anáilisis de datos, que arroja resultados favorables que permiten establecer avances favorables para la competitividad del sector con la afectación de las políticas públicas emanadas durante el período analizado.

Palabras clave: indicadores financieros, competitividad, políticas públicas, economía.

\begin{abstract}
This research project aims to contribute to the analysis of the progress in competitiveness and financial performance of the Colombian sugar sector during the period from 2013 to 2016, in contrast to public policies issued in commercial, labor and fiscal matters and the possible effects for its performance It is based on the theories of resources and capabilities and from corporate finance with financial indicators of profitability to measure business effectiveness by associating the management of costs and expenses in the generation of benefits. The research is descriptive, longitudinal, and inductive and uses the SAS statistical software for data analysis, which yields favorable results that allow establishing favorable progress for the competitiveness of the sector with the impact of public policies emanating during the period analyzed.
\end{abstract}

Keywords: Financial Indicators, Competitiveness, Public Politics, Economy. 


\section{INTRODUCCIÓN}

Durante el siglo XXI en Colombia se han establecido continuos cambios desde las políticas públicas en materia comercial, laboral y tributario, orientados al fortalecimiento económico y social del país y con la pretención de avanzar en el logro de mayores estándares de competitividad, generando afectaciones al desempeño empresarial.

En materia laboral, se resaltan la Ley 1429 denominada como la "Ley para la formalización y generación de empleo", propuso incentivos para la formalización de las empresas en las etapas iniciales orientados a aumentar los beneficios y disminuir los costos de formalizarse mediante la progresividad en los impuestos y contribuciones, como para el caso del impuesto de renta, industria y comercio, contribuciones a las Cámaras de Comercio por la inscripción y/o renovación del registro mercantil, aportes parafiscales y a la seguridad social de sus empleados. La Ley 1610 de enero 2 de 2013, se orientó a la regulación de algunos aspectos sobre las inspecciones del trabajo y los acuerdos de formalización laboral.

En lo comercial, el Decreto 019 del 10 de enero de 2012, orientado a suprimir o reformar regulaciones, procedimientos y trámites innecesarios existentes en la Administración Pública, al cual se le denominó “Ley Anti tramite". Entre muchos aspectos, simplifica una serie de procedimientos con el objeto de facilitar la actividad de las personas naturales y jurídicas ante las autoridades que cumplen funciones administrativas y así contribuir a la eficacia y eficiencia de autoridades gubernamentales y sector empresarial.

En el año 2009 se emana la Ley 1314 que establece el proceso de convergencia en Colombia hacia las Normas Internacionales de Información Financiera, que corresponde a estándares de contabilidad y aseguramiento de la información reconocidos internacionalmente. Esta Ley tuvo su impacto a partir del año 2014 cuando se establecen los decretos reglamentarios para el proceso de convergencia desarrollado entre los años 2014 a 2016, este cambio de las normas contables generó afectaciones financieras. De acuerdo con lo anterior, para el período comprendido entre 2013 a 2016 se expiden el Decreto 3022 en 2013, Decreto 2420 de 2015, Decreto 2496 de 2015, Decreto 2101 de 2016, Decreto 2131 de 2016 y Decreto 2132 de 2016. 
En lo fiscal, la Ley 1430 de diciembre 29 de 2010 elimina la deducción en renta por inversión, propone la eliminación progresiva al gravamen a los movimientos financieros, fiscalización al recaudo del IVA, propone la retención a pago de intereses de crédito externo, elimina la sobretasa del $20 \%$ al consumo de energía eléctrica al sector industrial y establece reformas arancelarias.

Ley 1607 de diciembre 26 de 2012, las cuales, sobre todo la última, han determinado una serie de cambios como la reagrupación y reasignación de tarifas para el caso del IVA, disminución del impuesto de renta del $33 \%$ al $25 \%$ para personas jurídicas. Se crean nuevos impuestos como el CREE o impuesto sobre la renta para la Equidad con tarifa del 8 \% e incremento para los siguientes años al 9 \%; el Impuesto Mínimo Alternativo (IMAN) e Impuesto Mínimo Alternativo Simple (IMAS). Igualmente, se crea un impuesto nacional al consumo respecto de algunos bienes y servicios, como telefonía al $4 \%$, restaurantes al $8 \%$ que es excluido del IVA y para vehículos, a tarifas del 8 y $16 \%$ (García y García, 2018).

Ley 1739 de 2014 mantuvo el gravamen a los movimientos financieros (GMF) con una tarifa del 4 por mil hasta el año 2018, para iniciar su desmonte gradual a partir de 2019. Creación del impuesto a la riqueza que recae sobre el patrimonio líquido al 1 de enero del año 2015, igual o superior a $\$ 1000$ millones, para los contribuyentes del impuesto sobre la renta y personas naturales y jurídicas extranjeras que posean riqueza en el país directa o indirectamente a través de establecimientos permanentes. Se modifica el IMAS y se crea sobretasa al CREE cuando la base gravable del impuesto supere los $\$ 800$ millones, el pago de la sobretasa se debe hacer por anticipado en dos cuotas anuales con base en la base gravable del período inmediatamente anterior. Este tributo tendrá aplicación desde 2015 hasta 2018. La base gravable del CREE no podrá ser inferior al $3 \%$ del patrimonio líquido del contribuyente en el último día del año gravable inmediatamente anterior. Se mantiene la prohibición de compensar el CREE con otros impuestos.

La Ley 1819 de 2016 establece la tarifa general del 33 \% para impuesto de renta a personas jurídicas y la depuración del impuesto de renta por sistema cedular, establece la tarifa general del IVA del $19 \%$, establece el pago del IVA por período bimestral o cuatrimestral, establece el monotributo como opción para la declaración de renta 
Políticas públicas orientados al logro de mayores niveles de competitividad; y como bien lo menciona Porter (1990), "Empresas Competitivas" conducen a "Naciones competitivas", según el Reporte Mundial de Competitividad logrado en el Foro Económico Mundial (WEF, por su sigla en inglés) en Suiza, para el período 2012-2013, las "empresas u organizaciones" son presentadas como el primer factor que impulsan las economías hacia el logro de mayores niveles de competitividad.

De acuerdo con el Índice Global de Competitividad (IGC) los doce pilares en los cuales se soporta el desarrollo de la competitividad a nivel global, se presentan en primer lugar a las "instituciones" como soporte de las economías de los países; las cuales, entre otras, se ven afectadas por la normatividad legal, regulación y cargas impositivas propias de cada país, que aporta o inciden positiva o negativamente para el logro de mayores niveles de competitividad en un entorno globalizado. En el segundo grupo y como séptimo pilar se encuentra la eficiencia del mercado laboral en el cual la normatividad y cargas impositivas inciden de manera importante para su desarrollo (IGC, 2016).

Por su parte, para el año 2005, el sector azucarero colombiano se consolida como un complejo productivo en transición, que asume ir a la par con las exigencias de este sector en el ámbito mundial, e inicia un proceso de redireccionamiento estratégico basado en la agregación de valor para sus productos y en la diversificación de su portafolio de productos; el cual, hasta ese momento tenía como protagonista un único producto: El azúcar. Es así como se inicia para la conocida zona de agricultura especializada en la caña de azúcar del país, la producción de Alcohol Carburante o Etanol a partir de la caña de azúcar; además, proyecta continuar con la cogeneración de energía a partir de los desperdicios del proceso productivo del azúcar, entre otros. La diversificación permitiría mitigar el riesgo de competir con un producto en un ambiente altamente competitivo a nivel mundial, en el cual, Colombia es reconocida como el país con mayor productividad en la obtención de caña de azúcar por hectárea, pero con costos de producción superiores a los de los sus principales competidores internacionales como Brasil, que finalmente por cubrir el $46 \%$ de la demanda mundial de azúcar, es quien marca el rumbo de los precios internacionales del producto; y es así como se da inicio entonces al nacimiento y desarrollo de las empresas de Biocombustibles en Colombia y en el mundo (Ascaña, 2012).

En la tabla 1 se presentan cifras del sector azucarero en el periodo 2014-2016, que ratifican la importancia económica y social de la industria azucarera para la región que impacta y para el país. 
Tabla 1. Cifras importantes del sector azucarero, 2014-2016.

\begin{tabular}{|c|c|c|c|c|}
\hline Variable & 2013 & 2014 & 2015 & 2016 \\
\hline $\begin{array}{l}2018 \text { área sembrada } \\
\text { en caña de azúcar }\end{array}$ & 205.456 & 230.303 has & 232.030 has & 238.204 has \\
\hline $\begin{array}{c}\text { Propiedad de la } \\
\text { tierra }\end{array}$ & ni & $\begin{array}{c}75 \% \text { pertenece } \\
\text { a más de } 2750 \\
\text { proveedores de caña. } \\
\text { 25\% pertenece a } 13 \\
\text { ingenios }\end{array}$ & $\begin{array}{c}75 \% \text { pertenece a más } \\
\text { de } 2750 \text { proveedores de } \\
\text { caña. } 25 \% \text { pertenece a } \\
15 \text { ingenios }\end{array}$ & $\begin{array}{c}75 \% \text { pertenece a más } \\
\text { de } 2750 \text { proveedores de } \\
\text { caña. } 25 \% \text { pertenece a } \\
14 \text { ingenios }\end{array}$ \\
\hline $\begin{array}{l}\text { Tamaño promedio } \\
\text { de la propiedad }\end{array}$ & ni & $\begin{array}{c}60 \text { has. } 65 \% \text { de las } \\
\text { fincas tiene menos } \\
\text { de } 60 \text { has }\end{array}$ & $\begin{array}{c}62.2 \text { has. } 65 \% \text { de las } \\
\text { fincas tiene menos de } \\
60 \text { has }\end{array}$ & $\begin{array}{c}63 \text { hectáreas. } 659 \text { de las } \\
\text { fincas tiene menos de } \\
60 \text { has }\end{array}$ \\
\hline $\begin{array}{l}\text { Producción de } \\
\text { azúcar }\end{array}$ & 2.127 .000 & 2.396.000 ton & 2.354.000 ton & 2.091 .000 \\
\hline $\begin{array}{l}\text { Ventas de azúcar } \\
\text { en Colombia }\end{array}$ & 1.407 .000 & 1.600.000 ton & 1.648.000 ton & 1.567.000 ton \\
\hline $\begin{array}{l}\text { Exportaciones } \\
\text { de azúcar }\end{array}$ & 719.000 & 796.000 ton & 725.000Toneladas & 518.000 ton \\
\hline $\begin{array}{l}\text { Valor de las } \\
\text { exportaciones }\end{array}$ & $\begin{array}{l}\text { 125.681.200 } \\
\text { dólares }\end{array}$ & 376.000 .000 dólares & 313.000.000 dólares & 272.000 .000 \\
\hline $\begin{array}{l}\text { Empleos directos } \\
\text { e indirectos } \\
\text { generados (según } \\
\text { FINAGRO) }\end{array}$ & 217.477 & 225.749 & 228.935 & 229.620 \\
\hline $\begin{array}{c}\text { Producción de Bio } \\
\text { Etanol }\end{array}$ & $\begin{array}{l}387.859 \text { litros } \\
\text { en } 5 \text { destilerías }\end{array}$ & $\begin{array}{c}406.000 .000 \text { litros en } \\
5 \text { destilerías }\end{array}$ & $\begin{array}{c}457.000 .000 \text { litros en } 6 \\
\text { destilerías }\end{array}$ & $\begin{array}{c}434.000 .000 \text { litros en } 6 \\
\text { destilerías }\end{array}$ \\
\hline $\begin{array}{l}\text { Porcentaje de } \\
\text { reducción de } \\
\text { emisiones de gases } \\
\text { efecto invernadero }\end{array}$ & $\begin{array}{l}74 \% \text { con } \\
\text { respecto al } \\
\text { combustible } \\
\text { fósil }\end{array}$ & $\begin{array}{l}74 \% \text { con respecto al } \\
\text { combustible fósil }\end{array}$ & $\begin{array}{l}74 \% \text { con respecto al } \\
\text { combustible fósil }\end{array}$ & $\begin{array}{l}74 \% \text { con respecto al } \\
\text { combustible fósil }\end{array}$ \\
\hline $\begin{array}{c}\text { Cogeneración de } \\
\text { energía }\end{array}$ & ni & $\begin{array}{l}1297 \text { GWh en } 12 \\
\text { plantas }\end{array}$ & 1381 GWh en 12 plantas & 1417 GWh \\
\hline $\begin{array}{l}\text { Aporte de energía } \\
\text { al Sistema } \\
\text { Interconectado } \\
\text { Nacional }\end{array}$ & ni & $442 \mathrm{GWh}$ & 514 GWh & $593 \mathrm{GWh}$ \\
\hline Contribución al PIB & $0,36 \%$ & ni & $0,50 \%$ & $0,70 \%$ \\
\hline
\end{tabular}

Fuente: elaboración propia adaptado de Asocaña, informes azucareros de los años 2013, 2014, 2015, 2016 
Así mismo, en la tabla 2 la evolución de los precios del azúcar y del alcohol carburante entre los años 2012 y 2016, donde se aprecia que la actividad comercial está sometida a la volatilidad de los precios internacionales del azúcar y a los cambios en la tasa de cambio del dólar, que incidió en la caída constante del precio del azúcar entre los años 2013 a 2015, con una tasa de cambio igualmente baja, la cual tuvo un incremento significativo en los años 2015 y 2016, lo que ciertamente beneficio las exportaciones del azúcar (Procaña, 2017).

Tabla 2. Variables comerciales representativas del azúcar, años 2012 - 2016.

\begin{tabular}{|c|c|c|c|c|c|}
\hline Promedio año & $\mathbf{2 0 1 2}$ & $\mathbf{2 0 1 3}$ & $\mathbf{2 0 1 4}$ & $\mathbf{2 0 1 5}$ & $\mathbf{2 0 1 6}$ \\
\hline $\begin{array}{c}\text { Libra azúcar NY } \\
\text { (ctvs dólar) }\end{array}$ & 21,58 & 17,48 & 16,34 & 13,14 & 18,14 \\
\hline $\begin{array}{c}\text { Ton azúcar Londres } \\
\text { (US\$/Ton) }\end{array}$ & 588 & 491 & 440 & 374 & 449 \\
\hline $\begin{array}{c}\text { Kg azúcar Nacional } \\
\text { (\$) }\end{array}$ & 1.360 & 1.247 & 1.335 & 1.712 & 2.197 \\
\hline $\begin{array}{c}\text { TRM } \\
\text { (\$) }\end{array}$ & 1.798 & 1.869 & 2.001 & 2.742 & 3.055 \\
\hline $\begin{array}{c}\text { Exportación azúcar } \\
\text { Alcohol carburante } \\
\text { \$/Litro }\end{array}$ & $51 \%$ & $48 \%$ & $53 \%$ & $57 \%$ & $55 \%$ \\
\hline
\end{tabular}

Fuente: elaboración propia adaptado de, Procaña (2017). Sector agroindustrial de la caña de azúcar

Es importante resaltar la posición que ostenta Colombia en el indicador de productividad de azúcar a nivel mundial entre los años 2011 y 2015, ocupa el primer lugar con una producción de 15,5 toneladas de azúcar por hectárea. Brasil, que es el primer productor de azúcar a nivel mundial, ocupa el quinto lugar con una producción de 10,7 toneladas por hectárea. Podría pensarse que este país podría aprovechar la economía de escala, pero no es así. Colombia lo supera por su permanente programa de

Es innegable la importancia que tiene el sector azucarero en la composición del PIB nacional y de los departamentos donde actúa. En la Tabla 3, se observa que, en el año 2015, aportó al PIB nacional con $0,7 \%$ y lo significativo que es en los departamentos del Valle, Cauca y Risaralda. Es de resaltar la importancia que tiene en el Cauca con casi una décima parte del PIB del departamento y en el Valle aporta más de la tercera parte del PIB agrícola del departamento. 
Tabla 3. Participación del sector azucarero en el PIN nacional y local - 2015.

\begin{tabular}{|l|c|c|c|}
\hline & PIB Agrícola \% & PIB Industrial \% & PIB Total \% \\
\hline Colombia & 3,7 & 2,7 & 0,7 \\
\hline Risaralda & 2,2 & 10,7 & 2,7 \\
\hline Valle de Cauca & 38,7 & 14,4 & 5,4 \\
\hline Cauca & 25,3 & 25,8 & 9 \\
\hline
\end{tabular}

Fuente: elaboración propia adaptado de Asocaña (2017) Sector azucarero colombiano 2016-2017

De acuerdo con lo anterior, es imperativo establecer las afectaciones al desempeño financiero y competitivo del sector azucarero colombiano respecto de los cambios en las políticas públicas orientadas a avanzar hacia el logro de mayores niveles de competitividad para el país y sus organizaciones, que permita establecer ¿Cuáles son las afectaciones financieras y competitivas para el sector azucarero colombiano respecto de los cambios en la normatividad comercial, fiscal y laboral del año 2013 a $2016 ?$

Para establecer las afectacioones financieras y competitivas se aborda desde la teoría financiera el análisis mediante indicadores financieros, que se correlaciona con la teoría de recursos y capacidades para el análisis de la competividad.

Análisis financiero. De acuerdo con Hernández (2005) mediante el análisis financiero se puede establecer el desempeño de una empresa a partir de la información financiera histórica generada en su desarrollo operativo a la cual se le aplica un análisis para determinar el desempeño financiero pasado, establecer la situación financiera actual de la empresa y fundamentar el proceso de toma de decisiones que generará impactos futuros.

Según Rubio (como se citó en Nava y Marbellis, 2009) en el desarrollo del análisis financiero se aplican una serie de técnicas a partir de la información financiera generada por las organizaciones, para el establecimiento de medidas útiles para diagnosticar el desempeño financiero de la empresa y fundamental en el proceso de toma de decisiones. 
Por su parte, Brigham y Houston (Como se citó en Sepúlveda, Moscoso y Restrepo, 2017) señalan que un análisis financiero integral considera afectaciones externas y el desempeño financiero de la empresa que identifica como factores internos.

El análisis interno comprende el estudio de la información obtenida a partir de los estados financieros e información cualitativa como bases estratégicas, ventajas competitivas, tecnología utilizada, estructura de la organización, entre otros; de otra parte, el análisis externo se alimenta de información respecto al entorno y perspectivas macroeconómicas, así como la situación política y social del país, que permitirá obtener un análisis global de la situación de la empresa. (p.137).

De acuerdo con lo anterior, el proceso de análisis o diagnóstico financieros además de analizar las cifras de los estados financieros de la empresa analizada se complementa con el análisis con información básica interna de tipo cualitativo y con el análisis del entorno en el cual se desarrolla la empresa o factores externos, estableciéndose como fundamentales en el desempeño de las organizaciones (Ortiz, 2015).

Se establecen varios grupos de indicadores financieros orientados a determinar la salud financiera de las empresas en términos de liquidez, endeudamiento, rentabilidad y gestión; los cuales se calculan a partir de las cifras del estado de situación financiera y del estado de resultados, según cada caso (Ortiz, 2015).

Ortiz (2015) propone establecer un adecuado conjunto de indicadores financieros que permitan determinar la salud financiera de la empresa analizada y que puede servir como referente para las demás empresas pertenecientes al mismo sector; pero también, de acuerdo con el objetivo del análisis se deberán seleccionar los indicadores financieros relevantes que aporten al propósito establecido para cada análisis.

Los indicadores financieros de rentabilidad, que como lo señala Ortiz (2015), también se conocen como indicadores de lucro indicadores de rendimiento, permiten "...medir la efectividad de la administración de la empresa para controlar los costos y gastos y de esta manera convertir las ventas en utilidades" (Ortiz, 2015, p.201). 
En la tabla 4 se presentan los indicadores financieros de rentabilidad que relacionan la efectividad en la gestión financiera de los costos respecto de las ventas para la efectiva generación de utilidades, y los resultados de su cálculo se establecen porcentualmente.

Tabla 4. Indicadores financieros de rentabilidad.

\begin{tabular}{|c|c|c|}
\hline Nombre del indicador & Fórmula & Detalle \\
\hline Margen Bruto de Utilidad & $\frac{\text { Utilidad Bruta }}{\text { Ventas Netas }}$ & $\begin{array}{l}\text { Por estar determinada la utilidad bruta por } \\
\text { las ventas menos el costo de ventas, se } \\
\text { considera para el caso de las empresas } \\
\text { industriales el impacto del costo de } \\
\text { ventas que está representado por todos } \\
\text { los costos de producción asociados a las } \\
\text { ventas realizadas. }\end{array}$ \\
\hline Margen Operacional de Utilidad & $\frac{\text { Utilidad Operacional }}{\text { Ventas Netas }}$ & $\begin{array}{l}\text { Indica si el negocio es lucrativo o no, } \\
\text { pues además de considerar el costo de } \\
\text { ventas, también considera los gastos } \\
\text { operacionales de administración y los } \\
\text { gastos operacionales de ventas para la } \\
\text { determinación de la utilidad operacional. }\end{array}$ \\
\hline Margen Neto de Utilidad & $\begin{array}{l}\text { Utilidad Neta } \\
\text { Ventas Netas }\end{array}$ & $\begin{array}{l}\text { Está afectado por el costo de ventas, los } \\
\text { gastos operacionales de administración y } \\
\text { de ventas, y adicionalmente está afectado } \\
\text { por ingresos no operacionales, egresos } \\
\text { no operacionales y por el impuesto de } \\
\text { renta. }\end{array}$ \\
\hline
\end{tabular}

Fuente: elaboración propia a partir de Ortiz (2015)

Así mismo, los indicadores financieros de las empresas permiten aportar en la determinación de los indicadores financieros del sector de la economía al que pertenecen, lo cual aporta significativamente para el análisis financiero del desempeño del sector.

Competitividad. Siguiendo a Porter (1980), la competitividad, expresada en términos de productividad, es la que les da ventaja competitiva a las empresas o a los sectores empresariales, quienes dinamizan todos sus recursos, experiencias, conocimientos y atributos que los hacen diferentes o únicos y, por supuesto, los hace mejores que a sus competidores. Lo ideal es que esta ventaja competitiva, no sea fácilmente alcanzada, imitada o superada por los competidores 
y que sea sostenible en el largo plazo. Las organizaciones que no puedan sostener este ritmo competitivo no tendrán los resultados para generar valor por encima de la media de su sector y tenderán a tener pérdidas y salir del mercado. (Avalos, 2009)

Teoría de los recursos y capacidades. El enfoque de los recursos y capacidades considera dos lecturas: lo estratégico y lo económico. En el plano económico, hace énfasis en conseguir el beneficio a largo plazo, porque éste se conserva a pesar de la competencia, pero si determinan la ventaja competitiva de manera sustentable. Los beneficios a largo plazo se pueden mantener si se tienen un producto homogéneo, existe libre movilidad de los recursos y se cuenta con información perfecta (Fong, 2005), aspecto poco probable porque la incertidumbre siempre está rondando en torno a la organización.

La lectura estratégica se basa en abordar la organización observando sus recursos y capacidades y su incidencia en la rentabilidad. Esta lenguaje o lectura brinda un enfoque teórico - metodológico que permite encontrar estrategias pertinentes a los objetivos organizacionales (Saéz, 2000). La ruta para conseguir la ventaja competitiva de la organización orienta a que se potencialicen todos los recursos y capacidades que posee la empresa para ser superior a la competencia. Es así como la competitividad se consigue por la manera en que se aplican los componentes propios (endógenos) de la organización para conseguir mayor cantidad de producción o de servicio, mejor calidad y no por las condiciones del entorno (exógenas), (Cardona, 2011). Estas condiciones endógenas son las que hacen que unas empresas se diferencien de otras, que las haga únicas e inimitables, que unas tengan ventajas competitivas por la gracia de su estrategia. Al fin y al cabo, las reglas de juego del contexto social, político, económico y normativas son las mismas para todos los competidores, bueno, al menos en teoría.

En la tabla 5, se presentan de manera sintética los recursos que se deben tener en cuenta a la hora de estudiar la competitividad de una empresa o un conglomerado. 
Tabla 5. Recursos de la empresa

\begin{abstract}
Recursos: Activos físicos bajo el servicio y control de la empresa para desarrollar su estrategia de competencia. Incluye activos, capacidades, proceso organizativo, característica empresarial, información y conocimiento (Grant, 2006).

Para Wernerfelt (1984), son fortaleza y/o debilidad de activos tangibles e intangibles que están semipermanentemente en la firma, como: marca, conocimiento de tecnología interna, habilidad de las personas, contactos, maquinaria, eficiencia de los procesos, capital, entre otros.
\end{abstract}

\begin{tabular}{|c|c|c|}
\hline & $\begin{array}{l}\text { Recursos tangibles: Son materiales, } \\
\text { cuantificables, medibles y tienen soporte } \\
\text { físico. (Weston, 1994). }\end{array}$ & $\begin{array}{l}\text { Recursos intangibles: información interna y externa. } \\
\text { Formación, conocimiento y experiencia del talento } \\
\text { humano tangible. (Barney, 1991; Grant, 2006). }\end{array}$ \\
\hline Humanos & $\begin{array}{l}\text { Indican el personal en cuanto a su } \\
\text { cantidad, cualificación, jerarquía y } \\
\text { organización. }\end{array}$ & $\begin{array}{l}\text { Oficio, experiencia, habilidad, cultura, motivación, } \\
\text { compromiso. Forma de razonar y tomar decisiones. } \\
\text { Capacidad de aprendizaje, flexibilidad, adaptación, } \\
\text { trabajo en equipo, liderazgo, relaciones personales, } \\
\text { emprendimiento, entre otros. }\end{array}$ \\
\hline Físicos & $\begin{array}{l}\text { Planta y su distribución, procesos flexibles, } \\
\text { instalación, equipos y recursos logísticos. }\end{array}$ & \\
\hline Técnicos & Hardware & $\begin{array}{l}\text { Dominio de tecnologías, rapidez de respuesta a los } \\
\text { cambios mediante la innovación que dé respuesta } \\
\text { pertinente al mercado. }\end{array}$ \\
\hline Financieros & $\begin{array}{l}\text { Relación entre recursos económicos } \\
\text { propios y ajenos, capacidad de } \\
\text { endeudamiento y de generar recursos } \\
\text { monetarios. }\end{array}$ & $\begin{array}{l}\text { Capacidad de gestión efectiva de los recursos } \\
\text { financieros. }\end{array}$ \\
\hline
\end{tabular}

Fuente: elaboración propia a partir de Grant (2006), Wernerfelt (1984), Weston (1994) y Barney (1991)

Capacidades. Capacidades se consideran los conocimientos, habilidades y tecnologías que emergen del aprendizaje colectivo de la empresa a causa del uso combinado de recursos, las rutinas, el intercambio de la información que permanentemente está haciendo el talento humano de la organización, motivado por los incentivos y la interacción social (Cuervo, 1993). Sumado a lo anterior, se pueden considerar como la gestión de los recursos, al interior de la organización con el fin de sostener o acrecentar las capacidades de la empresa. Las capacidades son la forma en que se dinamizan los recursos y se ponen en interacción para que la empresa sea competitiva. En otras palabras, es el conocimiento acumulado y rutinas de trabajo del talento humano que permanentemente está gravitando en torno a los recursos para obtener su máximo potencial en favor de la competencia organizacional. 
Las capacidades se pueden entender como "competencias distintivas" para mencionar lo que la organización hace mejor que su competencia. Lo importante es distinguir aquellas que son esenciales para los objetivos de la organización y no distraerse en aquellas que son marginales. Se distinguen tres aspectos claves que deben cumplir dichas competencias: que el valor agregado sea identificado y altamente apreciado por los clientes, que sea totalmente diferenciador de la competencia y que se pueda aplicar a muchos productos de la compañía. (Suárez, J. Ibarra, S. s.f.)

Es importante identificar cuáles son las capacidades que están al interior de la organización, Grant (1991), indica que se deben de identificar y/o clasificar de acuerdo al área funcional de la organización donde se encuentren. Pero también, es importante identificar aquellas capacidades que emergen sinérgicamente de diferentes áreas funcionales. En cuanto a su medición, es conveniente hacerlo mediante el Benchmarking, para tener mayor objetividad. No tiene sentido compararse consigo mismo, una medida más objetiva es la calificación relativa que se tiene con respecto a la competencia. La idea es compararse con el mejor en su sector. Grant, complementa lo anterior afirmando que no basta con el "ensamblaje" mecánico de recursos, las capacidades implican gestión, coordinación, establecimiento de rutinas en todos los niveles y procesos, especialmente en los procesos previsibles y regulares, lo que constituye la esencia de las capacidades organizativas. (Suárez, J. Ibarra, S. s.f.)

\section{OBJETIVOS}

Establecer las afectaciones financieras y competitivas para el sector azucarero colombiano respecto de los cambios en la normatividad comercial, fiscal y laboral del año 2013 a 2016.

\section{OBJETIVOS ESPECÍFICOS}

- Identificar los cambios de las políticas públicas en materia laboral, comercial y tributaria del año 2013 a 2016 con afectaciones al sector azucarero colombiano.

- Establecer desde la información financiera del sector azucarero colombiano del año 2013 a 2016, los indicadores que aporten al análisis de su desempeño y competitividad.

- Contrastar los impactos financieros ocasionados al sector azucarero colombiano y su competitividad respecto de los cambios en la normatividad comercial, fiscal y laboral, del año 2013 a 2016. 


\section{METODOLOGÍA}

La investigación es descriptiva, longitudinal, aplica método inductivo y de enfoque mixto, que propone el análisis las afectaciones financieras y en la competitividad ocasionadas al sector azucarero colombiano en contraste con los cambios en la normatividad comercial, fiscal y laboral durante el período 2013 a 2016.

El sector azucarero colombiano para el período comprendido entre los años 2013 a 2016 estaba conformado por trece ingenios, de acuerdo con el directorio estadístico de empresas consultado en la plataforma del geoportal del DANE. Tres de los trece ingenios azucareros atravesaron situaciones atípicas para el período analizado, por estar en proceso de liquidación, o de nacimiento de un nuevo ingenio, o en proceso de reestructuración; por lo cual se desestimaron, quedando conformada la población objetivo y la muestra seleccionada por 10 ingenios azucareros, lo que representa una muestra no probabilística intencionada o de conveniencia, como se aprecia en la tabla 6 .

Tabla 6. Ingenios azucareros.

\begin{tabular}{|l|}
\hline Ingenios \\
\hline Ingenio Carmelita S.A. \\
Ingenio del Cauca S.A.S. \\
Manuelita S.A. \\
Ingenio María Luisa S.A. \\
Ingenio Pichichi S.A. \\
Ingenio Providencia S.A. \\
Ingenio Risaralda S.A. \\
Ingenio San Carlos S.A. \\
Ingenio Rio Paila Castilla S.A. \\
Ingenio Mayagüez S.A.
\end{tabular}

Fuente: elaboración propia

\section{RESULTADOS}

Para las variables seleccionadas se establecen las afectaciones en el desempeño financieras para el sector azucarero durante los años 2013 a 2016. 


\section{MARGEN BRUTO DE UTILIDAD (MUB)}

Para el año 2013, el margen de utilidad bruta media para las empresas del sector azucarero fue del $16 \%$, que implicó que por cada peso generado en ventas 84 centavos se destinan a cubrir los costos de las mercancías vendidas, dejando 16 centavos de utilidad bruta. Para el año 2014 el margen de utilidad bruta representó el $20 \%$ y para el año 2016 fue del 27 \% (ver figura 1).

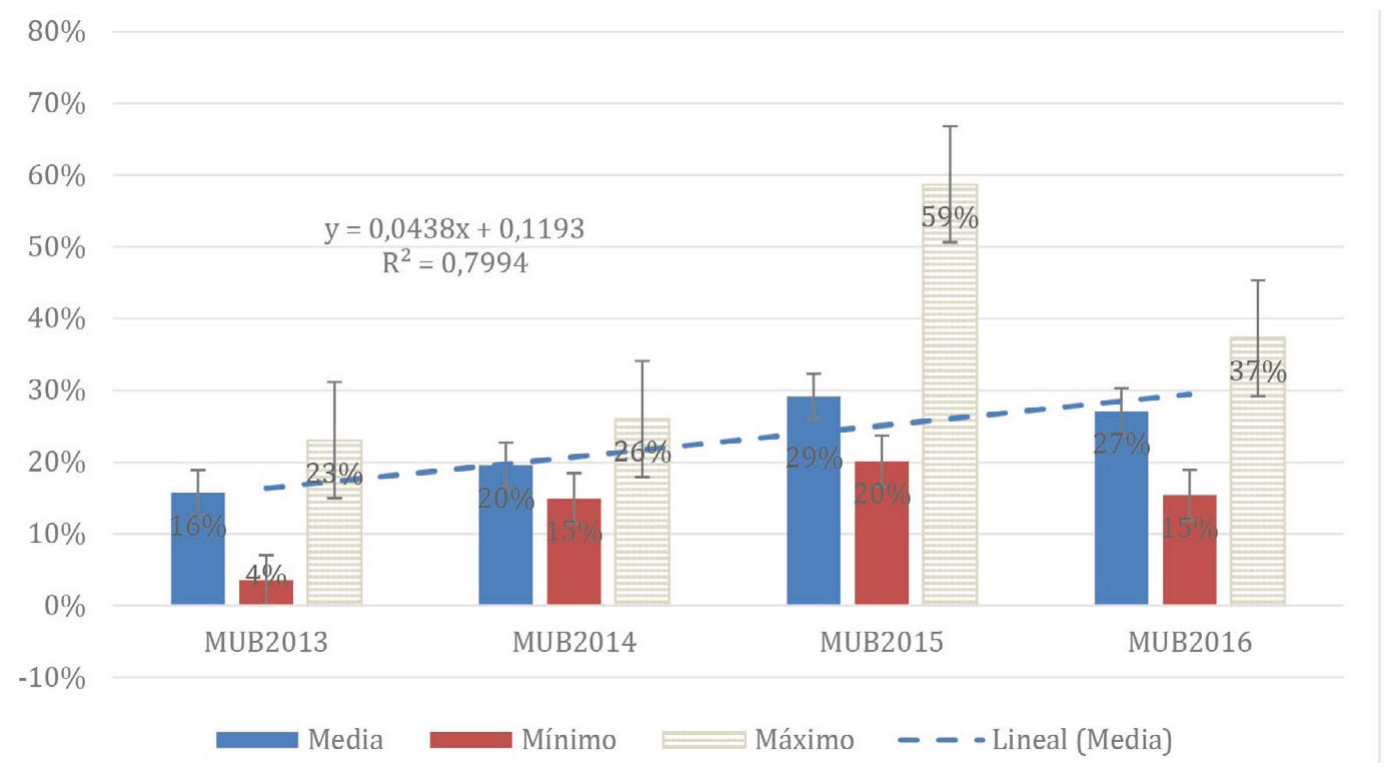

Figura 1. Margen Bruto de Utilidad 2013 a 2016.

Fuente: elaboración propia

Considerando que en el márgen utilidad bruta están considerados los costos de producción asociados a las ventas generadas, en los cuales están la mano de obra y recursos de producción, se puede establecer como el costo de ventas medio del año 2013 representó el 84 \% de las ventas, para el año 2014 se redujo al $80 \%$, para el año 2015 el $71 \%$ y para el año 2016 el $73 \%$, estableciéndose la disminución en los costos de producción para la generación de ventas que en el período analizado bajo del $84 \%$ al $73 \%$, se puede concluir un avance importante en la eficiencia del sector azucarero colombiano asociado a su productividad y pro lo tanto a su competitividad.

Por lo anterior, desde el margen de utilidad bruta se aprecia tendencia favorable y creciente, que implicó mejorar de índice del $16 \%$ en el año 2013 al $27 \%$ en el año 2016. Es importante resaltar que para el año 2015 se alcanzó el mayor nivel del margen de utilidad bruta con el $29 \%$. 
Para este indicador, se aprecia alto grado de concentración de los datos alrededor de la media, donde la varianza fluctúa entre 0.001 y 0.01 durante el período analizado; que indica que las empresas presentaron tendencias similares respecto del margen bruto de utilidad.

\section{MARGEN OPERACIONAL DE UTILIDAD (MUO)}

Para el año 2013, el margen de utilidad operacional medio para las empresas del sector azucarero fue del $2 \%$ que implica que por cada peso generado en ventas 98 centavos se destinan a cubrir los costos de las mercancías vendidas y los gastos de operación, dejando 2 centavos de utilidad bruta.

En el año 2014 el margen de utilidad operacional se incrementó al 7 \%; para el año 2015 el margen de utilidad bruta fue del $16 \%$ y del $18 \%$ en el año 2016. En el año 2015 se presenta la mayor variación de este indicador, que mostró incremento del 9\% respecto del año anterior.

Para este indicador, se aprecia alto grado de concentración de los datos alrededor de la media, donde la varianza fluctúa entre 0.005 y 0.007 durante el período analizado; que indica que las empresas presentaron tendencias similares respecto del margen de utilidad operativa.

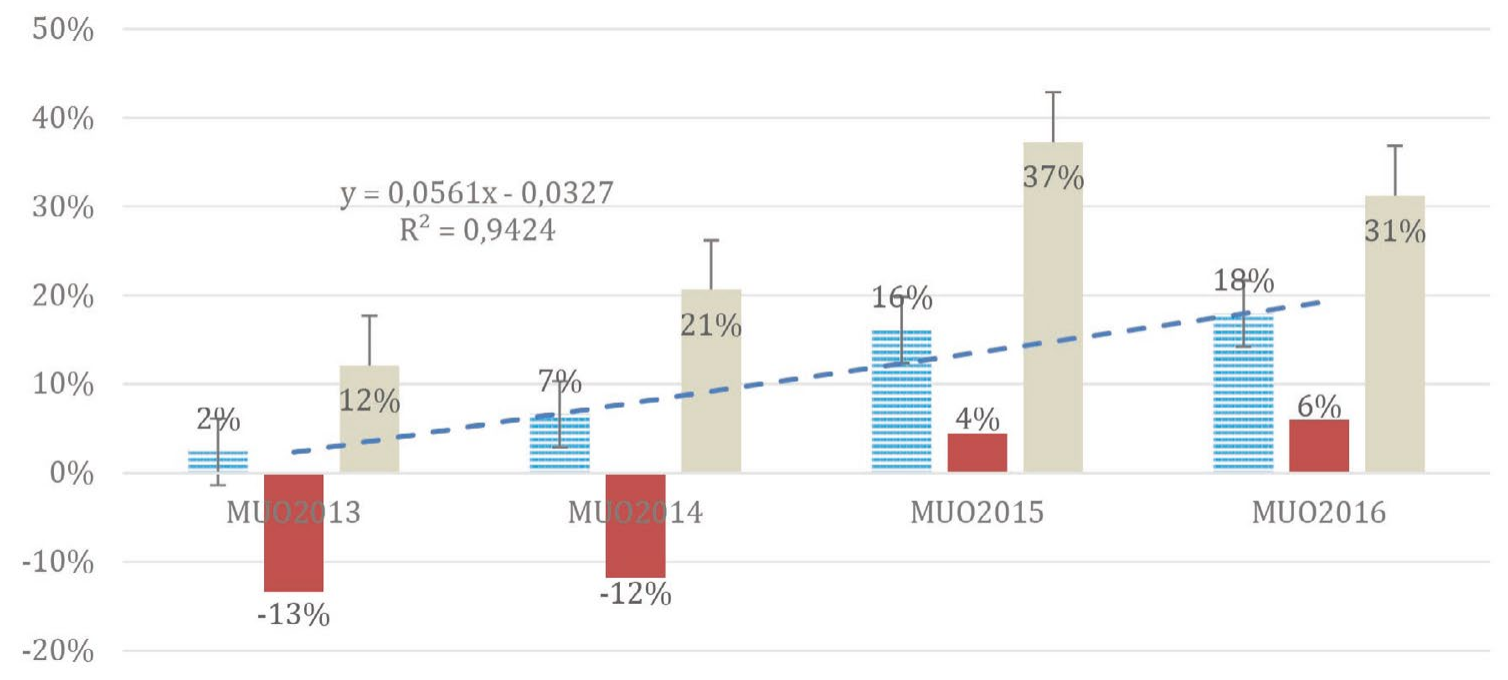

Figura 2. Margen Operacional de Utilidad 2013 a 2016

Fuente: elaboración propia 
Considerando que el margen de utilidad operacional está impactado por los costos de producción y los gastos operacionales de adminsitración y de ventas que fueron necesarios para la generación de ventas alcanzadas, donde en el año 2013 tanto costos como gastos medios representaron el 98 \% de las ventas, para el año 2014 representaron 93 \%, para el año 2015 el 84 \% y para el año 2016 el 82 \%, se puede concluir como para el período analizado se logro mayor productividad y avance en su competitividad al reducir del $93 \%$ al $82 \%$ costos y gastos del sector azucarero colombiano en la generación de ingresos en 11 \% durante el período analizado.

Se aprecia que el margen de utilidad operacional medio presenta una tendencia favorable y creciente, que implicó mejorar este indicador al pasar del 2 \% en el año 2013 hasta alcanzar el 18\% en el año 2016 gracias a la eficiencia en la gestión de sus recursos y capacidades de producción que en términos financieros se estabñecen a través de los costos de producción y los gastos operacionales.

\section{MARGEN NETO DE UTILIDAD (MUN)}

Para el año 2013, las empresas del sector azucarero presentaron pérdidas netas equivalentes al $0.4 \%$ sobre el valor de las ventas; lo anterior implica que, por cada peso que la empresa vendió, generó cuatro centavos adicionales de pérdidas; situación muy difícil para el fortalecimiento y crecimiento del sector azucarero colombiano (ver figura 3).

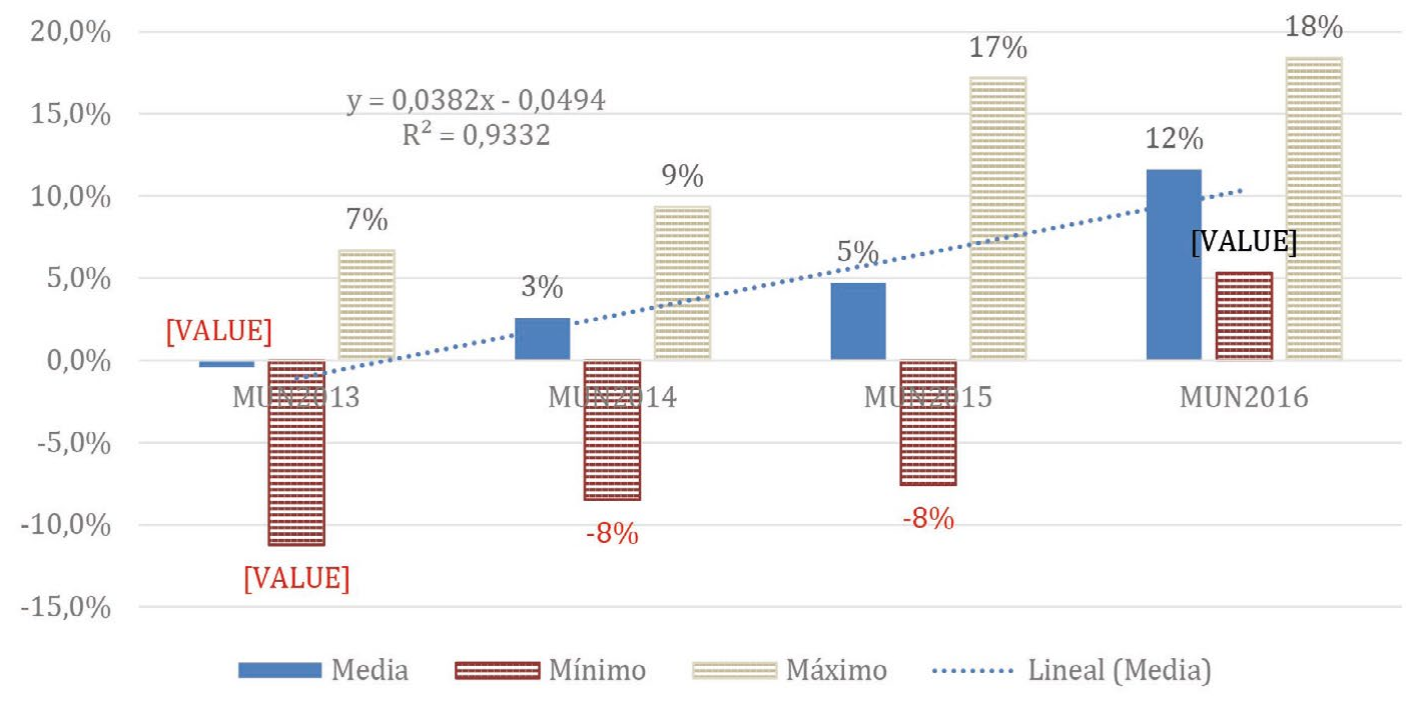

Figura 3. Margen Neto de Utilidad 2013 a 2016.

Fuente: elaboración propia 
Se aprecia que las empresas del sector azucarero pasan de pérdidas en el año 2013 a generar ganancias al año 2016, en una tendencia creciente positiva; que implica el mejoramiento de la gestión realizada por la empresa.

Para este indicador, se aprecia alto grado de concentración de los datos alrededor de la media, donde la varianza fluctúa entre 0.002 y 0.005 durante el período analizado; que indica que las empresas presentaron tendencias similares respecto del margen de utilidad neta.

Es importante considerar las afectaciones de los factores externos como los continuos cambios en las políticas públicas, especialmente en lo tributario y comercial dado el proceso de convergencia obligatorio en el reconocimiento contable financiero de los hechos económicos de las organizaciones por el paso de los PCGA a IFRS comprendidos entre 2014 a 2016; en contraste con los resultados en la gestión del sector azucarero que presentan un cambio importante en la tendencia de sus resultados asociados al uso de sus recursos, donde se pasa de tener pérdidas a obtener utilidades; demostrando mayor eficiencia en el desarrollo de su gestión y por lo tanto avances la competitivdad del sector. Para el año 2014 fue de 5 centavos y para el año 2016 de 12 centavos

\section{CONCLUSIONES}

Se establecen los hallazgos representativos identificados en el analisis realizado a partir de los enfoques aplicados desde la teória financiera y de recursos y capacidades.

\section{CON RESPECTO A LOS RECURSOS TANGIBLES}

Humanos. De acuerdo con los informes de sector azucarero, la industria ha tenido un sostenido crecimiento en el trabajo directo e indirecto generado. Es así como paso de 217.000 empleos en el año 2013 a 229.620 en el año 2016 (ver tabla 2). Según estos informes, el personal directo está contratado formalmente cumpliendo con todas las prestaciones de ley y la normatividad que en materia laboral ha expedido el gobierno en el periodo de estudio. Por el incremento sostenido que ha tenido en la producción, se puede inferir que es un personal que cumple con los perfiles, lo que hace que tenga la productividad mundial de azúcar más alta con una producción de 15.5 toneladas por hectárea de caña sembrada. 
Físicos. El sector azucarero, cuenta con una extensión de tierra considerable en el valle geográfico del rio Cauca. En el año 2013 contaba con 205.456 hectáreas para pasar a 238.204 hectáreas en el año 2016 (ver tabla 2). El 25\% de estas tierras son propiedad de los ingenios y el $75 \%$ son de los proveedores de caña. En el año 2016, el 65 \% de las fincas, posee menos de 63 hectáreas. Lo que hace que los terrenos no permitan labores de gran escala, haciendo subir los costos de las labores de campo.

El no tener un mayor porcentaje de la propiedad de la tierra, no significa una debilidad para el sector, dado que es una alianza de años que se ha venido consolidando por el cumplimiento en las obligaciones contraídas por parte de los ingenios. Por consiguiente, para poder trabajar estas grandes extensiones de tierra (pero fraccionadas) cuenta con maquinaria agrícola y de transporte, cuentan con modernas plantas de producción de azúcar y seis destilerías que producen alcohol carburante, para el cual tienen un mercado asegurado.

La productividad de estas plantas se expresa en una producción de azúcar a saber: en el año 2013 de 2.127 .000 toneladas, 2.396.000 en el 2014, 2.354.000 en el 2015 y 2.091 .000 en el año 2016. La producción de bioetanol en el año 2013 fue de 387.859 litros para pasar a 434.000 litros en el año 2016, año en que tuvo un leve descenso con respecto al año 2015. El sector también es cogenerador de energía, pasando de producir 1297 GWh en el año 2014 a 1417 GWh en el año 2016.

Financieros. En este punto, se analiza con respecto al Margen Bruto de Utilidad (MBU), Margen Operacional de Utilidad (MOU) y el Margen Neto de Utilidad (MNU), que fueron calculados por los investigadores del presente trabajo. Se observa como el MBU, pasa del 16 \% en el año 2013 al $27 \%$ en el año 2016. Hay que anotar el buen año que tuvo en el 2015 con MBU del $29 \%$, este indicador muestra un comportamiento con una pendiente positiva. Con respecto al MOU, inicia con un 2\% en el año 2013 (muy pobre) pero paulatinamente, va teniendo una recuperación hasta alcanzar el 18\% en el año 2016. El MNU, inicia con una actuación desastrosa en el año 2013, donde tiene pérdidas del $4 \%$ sobre las ventas. Pero durante el periodo de convergencia, IFRS, tiene una recuperación hasta obtener el 12\% en el año 2016.

En términos generales, el desempeño financiero del sector azucarero tuvo un significativo repunte durante el periodo 2013 a 2016. Al parecer, revisó los altos costos operativos que tenía en 
el año 2013 y se supo sobreponer en los años siguientes; lo cual se aprecia al reducir sus niveles de costos de ventas del $84 \%$ al $73 \%$, que representa una mejora de 11 puntos porcentuales, que aporta al fortalecimiento de la gestión operativa del sector azucarero colombiano y su competitividad. Lo cual se ratifica igualmente con el indicador de margen de utilidad operacional, donde se demuestra la reducción de costos de ventas y gastos operacionales del $98 \%$ al $82 \%$ que implica reducción de 16 puntos porcentuales, que implica mejoramiento de 11 puntos en relación de costos asociados con su proceso productivo y 5 puntos en la eficiencia de gastos asociados a su gestión de administración y de ventas.

Hay que tener presente que este avance en materia de competitividad fue logrado pese a las difíciles situaciones externas que debió enfrentar, como el precio del dólar que estuvo de capa caída en el periodo de estudio, especialmente en los años 2013 y 2014, mientras que en los años 2015 y 2016, tuvo un repunte; considrando que el precio de la divisa es representativo para el sector azucarero, dado que tiene un importante rublo en exportaciones. Además, el precio internacional del azúcar paso de 21, 58 centavos de dólar la libra en el año 2012 a 17,48 en el 2013; 16,34 en el $2014 ; 13,14$ en el 2015 y 18,14 en el año 2016.

Lo que explica el pobre desempeño del año 2013 (ver Tabla 3), pero se supo reponer ante precios bajos, especialmente en el 2014, lo que habla bien de la competitividad del sector. Además, se debe considrar que para el período analizado el impacto de reformas tributarias especialmente la Ley 1739 de 2014 y en materia comercial la convergencia de los principios de contabildiad generalmente aceptados en Colombia (PCGA) al acogimiento de las normas internacionales de información financiera (NIIF) establecidos a partir de la Ley 1314 de 2009 y reglamentada mediante Decreto 3022 en 2013, Decreto 2420 de 2015, Decreto 2496 de 2015, Decreto 2101 de 2016, Decreto 2131 de 2016 y Decreto 2132 de 2016.

\section{CON RESPECTO A LOS RECURSOS INTANGIBLES Y LAS CAPACIDADES}

Humanas. El hecho de que el sector azucarero haya conseguido la mayor productividad del mundo y esté creciendo en área cultivada de caña y por consiguiente en producción de azúcar, esté creciendo continuamente en la producción de bioetanol, significa que hay una gestión competitiva. Si se tiene en cuenta, que el recurso intangible es la capacidad de la organización para gestionar los recursos de la empresa, el sector azucarero lo está haciendo bien. Se evidencia que hay un 
"oficio" estratégico, táctico y operativo producto de la larga experiencia de este sector. Se evidencia la habilidad para superar impases como la caída de los precios internacionales del azúcar en simultánea con la caída de la divisa dólar. Hay una cultura organización que motiva y genera compromiso de todos los estamentos con los objetivos organizacionales. Existen aprendizajes y rutinas en las formas de hacer las cosas, incluso en la forma de razonar y tomar decisiones. La capacidad de aprendizaje les permite la flexibilidad para adaptarse a los continuos cambios que imponen las turbulencias de los contextos económicos, normativos y globales. Estas son las capacidades que desarrollan las organizaciones para ser cada vez más competitivas.

Técnicas. Están directamente relacionadas con las capacidades humanas, se refieren a lo que es capaz de hacer el talento humano de la organización. En el caso del sector azucarero, se trata del dominio que tienen las personas sobre la tecnología, la capacidad para dar respuestas oportunas y pertinentes a los cambios mediante la innovación. Es así como los ingenios están rodeados y acompañados por 6 organizaciones privadas claves como son: Asociación colombiana de productores y proveedores de caña de azúcar (PROCAÑA), Asociación de proveedores Ingenio Risaralda (AZUCARI), Asociación de cultivadores de caña de azúcar de Colombia (ASOCAÑA),

Centro de investigación de caña de azúcar de Colombia (CENICAÑA), Asociación colombiana de técnicos de la caña de azúcar (TECNICAÑA) y Comercializadora internacional de azúcares y mieles (CIAMSA). En estas organizaciones, están representados sus principales grupos de interés como son proveedores, comercializadores, cultivadores, técnicos e investigadores. Estos dos últimos le dan un gran valor intangible al sector por el conocimiento que genera para establecer rutinas de trabajo y desarrollar innovación clave para mantener la competitividad. Es decir, desarrollan la capacidad técnica del sector en toda su magnitud.

Financieros. Como ya se mencionó anteriormente, el sector azucarero supo sortear la baja en los precios internacionales del azúcar, la baja en el precio del dólar, lo que lleva a afirmar que ha desarrollado las capacidades para la gestión del recurso financiero. Ya en el año 2005, había demostrado su capacidad de buscar nuevos frentes de trabajo, cuando incursionó en la producción de biocombustibles, rubro de suma importancia en la productividad y por consiguiente en la competitividad del sector azucarero. Es de anotar que ha incursionado en otros sectores y en otras regiones dentro y fuera del país, gracias a su aprendizaje organizacional que sabe trans- 
ferir muy bien. En el periodo objeto de estudio, se observa como logro superar las dificultades presentadas en el año 2013 y sigue siendo un sector fuerte que jalona la competitividad de la región, pasando de aportar el 0,36 \% en el 2013 al 0,7 \% del PIB nacional en el 2016 (ver tabla 2), ciertamente, esto evidencia las capacidades en materia de gestión financiera, lo que le suma a la competitividad del sector.

Los ingenios que conforman el sector azucarero y el sector en sí tienen el control de sus factores endógenos, de tal manera que de ellos depende generar su ventaja competitiva. En la descripción de los recursos y capacidades, se puede observar como movilizan y gestionan sus recursos tangibles e intangibles para ser productivos y por consiguiente competitivos. Pese a tener la productividad más alta del mundo de toneladas de azúcar vs. Hectárea sembrada de caña, deben de trabajar más en el tema de los costos de producción que les restan productividad y por consiguiente afectan la competitividad. El sector cuenta con las habilidades y recursos para gestionar todos sus recursos, proponer y desarrollar estrategias y para ser innovadores.

Colombia cuenta con políticas e instituciones para el desarrollo de la competitividad y la productividad, las cuales están consignadas en el documento CONPES (2016). Está trabajando en infraestructura de vías y tecnológica de telecomunicaciones que se espera contribuyan a la competitividad de las empresas. Sin embardo, debe trabajar en recuperar la institucionalidad, acabar con la corrupción, avanzar en la calidad de la educación, disminuir la burocracia gubernamental ineficiente y sobre la complejidad de la norma fiscal (ver Tabla 1). Colombia, por supuesto, cuenta con un amplio abanico de normativas en el plano comercial, laboral e impositivo que se constituyen en un freno para la competitividad de las empresas y por consiguiente del país.

Finalmente, señalar que las condiciones favorables del contexto macroeconómico no necesariamente hacen que surjan espontaneaste empresas competitivas. Tampoco, el emprendimiento, la inversión y las ganas de hacer empresas, por sí solos, hacen que un país sea competitivo. La competitividad es el resultado del desarrollo de sinergias que se dan en la compleja relación e interacción entre el Estado y empresas.

Con los anañisis realizados se aprecia como para el sector azucarero colombiano el período comprendido entre 2013 a 2016 arroja un resultado favorable producto de su eficiente desempeño 
evidenciado en el control y disminución de los costos y gastos asociados a su proceso productivo y desempeño empresarial, demostrando avances importantes en el eficiente manejo de sus recursos y capacidades; en coherencia con los propósitos desde las políticas públicas orientadas a apoyar el desarrollo empresarial hacia el logro de mayores niveles de competitividad, que por los resultados establecidos se aprecian ambos, sector azucarero colombiano y políticas públicas, encaminados en un propósito compartido con resultados favorables.

\section{REFERENCIAS}

ASOCAÑA. (2016). Aspectos generales del sector azucarero colombiano 2015-2016. Recuperado de: https://www.asocana.org/documentos/2942016-24037160-00FF00,000A000,878787,C3C3C3, OFOF0F,B4B4B4,FF00FF,FFFFFF,2D2D2D,A3C4B5,D2D2D2.pdf

Avalos, F. (2009). Factores determinantes de la competitividad empresarial. Escuela de Postgrado UPC. Área de Investigaciones. Ciclo de conferencias doctorales para MBA-S.

Barney, J. B. (1991). Firm resources and sustained competitive advantage. Journal of Management, 17, 99-120.

Cardona, R. A. (2011). Estrategia basada en los recursos y capacidades, criterios de evaluación y el proceso de desarrollo. Revista Electrónica FORUM. Doctoral, 4. Mayo-Julio.

Congreso de la República de Colombia. (13 de julio de 2009). Regula principios y normas de contabilidad e información financiera y de aseguramiento de información aceptados en Colombia, se señalan las autoridades competentes, el procedimiento para su expedición y se determinan las entidades responsables de vigilar su cumplimiento. Ley 1314 de 2009.

Congreso de la República de Colombia. (29 de diciembre de 2010). Formalización y Generación de Empleo. Ley 1429 de 2010.

Congreso de la República de Colombia. (29 de diciembre de 2010). Normas tributarias de control y para la competitividad. Ley 1430 de 2010. 
Congreso de la República de Colombia. (16 de junio de 2011). Plan Nacional de Desarrollo, 2010-2014. Ley 1450 de 2011.

Congreso de la República de Colombia. (26 de diciembre de 2012). Normas en materia tributaria. Ley 1607 de 2012.

Congreso de la República de Colombia. (2 de enero de 2013). Regulación de algunos aspectos sobre las inspecciones del trabajo y lo acuerdos de formalización laboral. Ley 1610 de 2013.

Congreso de la República de Colombia. (23 de diciembre de 2014). Modificación del estatuto tributario, la ley 1607 de 2012, se crean mecanismos de lucha contra la evasión. Ley 1739 de 2014.

Congreso de la República de Colombia. (29 de diciembre de 2016). Reforma tributaria estructural, se fortalecen los mecanismos para la lucha contra la evasión y la elusión fiscal Ley 1819 de 2016.

Constitución Política de Colombia (1991). Constitución Política de Colombia.

Conpes. (2016). Política nacional de competitividad y productividad. Recuperado de: http://www.colombiacompetitiva.gov.co/sncei/Documents/Conpes-3527-de-2008.pdf

Cuervo, A. (1993). El papel de la empresa en la competitividad. Papeles de Economía Española, 56, 363-377.

Esser, K., Hillebrand, W., Messner, D. y Meyer, J. (1996). Competitividad Sistémica. Revista de la CEPAL, $59,39-52$.

Fong R, C. (2005). La teoría de los Recursos y Capacidades. Fundamentos microeconómicos. Producción académica de los miembros del sistema nacional de investigadores. Centro Universitario de las Ciencias Económicas Administrativas. Universidad de Guadalajara.

García, M. y García, T. (2018). Las reformas tributarias del milenio y su relación con los ingresos tributarios y la pobreza. Recuperado de: https://revistas.usantotomas.edu.co/index.php/iusta/article/ view/4646/html 
Grant, R.M. (2006). Dirección Estratégica: Conceptos, Técnicas y Aplicaciones. Madrid: Civitas.

Grant, R.M. (1991). The Resource - based theory of competitive advantage: Implications for Strategy Formulation. California Managem. Rev., Spring, 114 -135.

Membrillo, M. (2006). Desarrollo de una propuesta para la evaluación de la relación de actividades innovadoras con la competitividad de empresas farmacéuticas mexicanas. (Tesis). Facultad de Química, UNAM.

Ministerio de Industria y Turismo. (27 de diciembre de 2013). Marco técnico normativo para los preparadores de información financiera. Decreto 3022 en 2013.

Ministerio de Comercio Industria y Turismo. (22 de diciembre de 2016). Modificación parcial del marco técnico normativo de las Normas de Aseguramiento de la información, previsto en el artículo 1.2.1.1, Libro 1, Parte 2, Título 1, del Decreto 2420 de 2015. Decreto 2132 de 2016.

Naciones Unidas-Cepal (2001). Elementos de competitividad Sistémica de las Pequeñas y Medianas Empresas (PYME) del Istmo Centroamericano. México

Nava, R. Marbelis, A. (2009). Análisis financiero: una herramienta clave para una gestión financiera eficiente. RVG, 14), 606-628.

Ortiz, H. (2015). Análisis financiero aplicado y principios de administración financiera. Universidad Externado de Colombia. Bogotá, Colombia.

Porter, M. (1980). Competitive Strategy. Techniques for analizeng industries and competitors. New York: Free Press.

Presidencia de la Republica de Colombia. (10 de enero de 2012). Ley anti tramites. Decreto 019 de 2012. 
Presidencia de la Republica de Colombia. (10 de diciembre de 2015). Decreto Único Reglamentario de las Normas de Contabilidad, de Información Financiera y de Aseguramiento de la Información. Decreto 2420 de 2015.

Presidencia de la Republica de Colombia. (23 de diciembre de 2015). Modificación del decreto 2420. Decreto 2496 de 2015.

Presidencia de la Republica de Colombia. (22 de diciembre de 2016). Normas de Información Financiera para Entidades que no Cumplen la Hipótesis de Negocio en Marcha, a la Parte 1 del Libro 1 del Decreto 2420 de 2015, Único Reglamentario de las Normas de Contabilidad, Información Financiera y de Aseguramiento de la Información. Decreto 2101 de 2016.

Presidencia de la Republica de Colombia. (22 de diciembre de 2016). Modificación parcial del Decreto 2420 de 2015 modificado por el Decreto 2496 de 2015. Decreto 2131 de 2016.

Real Academia Española. (2019). Normatividad. Recuperado de https://dle.rae.es/srv/search?m=30\&w= normativo

SÁEZ de Viteri Arranz, D. (2000). El potencial competitivo de la empresa: recursos, capacidades, rutinas, y procesos de valor añadido. Investigaciones Europeas de Dirección y Economía de la Empresa, 6(3), 71-86.

Schwab, K. and World Economic Forum. (2016). The Global Competitiveness Report 2015 -2016. Retrieved from: http://www3.weforum.org/docs/gcr/2015-2016/Global_Competitiveness_Report_2 015-2016.pdf

Sepúlveda Rivillas, C. I; Moscoso Escobar, J. y Restrepo Londoño, A. L. (2017). Diagnóstico Financiero de las empresas en etapa temprana en Antioquia, 15(26), 131-155.

Suárez, J. y Ibarra, S. (s.f.). La teoría de los recursos y las capacidades. Un enfoque actual de la estrategia empresarial. Recuperado de https://dialnet.unirioja.es/descarga/articulo/793552.pdf 
Torres-Melo, J. y Santander, J. (2013). Introducción a las políticas públicas. Conceptos y herramientas desde la relación entre Estado y ciudadanía. Recuperado de https://www.funcionpublica.gov.co/ eva/admon/files/empresas/ZW1wcmVzYV83Ng==/imgproductos/1450056996_ce38e6d218235a c89d6c8a14907a5a9c.pdf

WEF. Schwab, K. (2010). The global competitiveness report 2009-2010. Retrieved from: http://www3. weforum.org/docs/WEF_GlobalCompetitivenessReport_2009-10.pdf

Wernerfelt, B. (1984). A, Resource-Based View ofthe Firm. Strategic Management Jounal, 5, 171-180.

Weston, J. y Brigham, E. (1994). Fundamentos de administración financiera. México: Mc Graw Hill, 1148 p. 